\title{
Análisis de la Sentencia n ${ }^{\circ}$ 16/21, de 15 de enero, de la Sección Segunda de la Audiencia Provincial de Valencia. El corte estético de orejas a los perros y su consideración como delito de maltrato animal en España
}

Eduardo Olmedo de la Calle

Doctor en Derecho

Fiscal de medio ambiente en la Fiscalía Provincial de Valencia

Recepción: Abril 2021

Aceptación: Junio 2021

Cita recomendada. OLMEDO DE LA CALLE, E., Análisis de la Sentencia nº 16/21, de 15 de enero, de la Sección Segunda de la Audiencia Provincial de Valencia. El corte estético de orejas a los perros y su consideración como delito de maltrato animal en España, dA. Derecho Animal (Forum of Animal Law Studies) 12/2 (2021). - DOI https://doi.org/10.5565/rev/da.574

\section{Resumen}

Se analizan en el artículo los requisitos jurisprudenciales que precisa el concepto de menoscabo grave de la salud del animal, en el sentido exigido por la STS 185/20, de 20 de mayo. Dichos requisitos se concretan en la necesidad de un tratamiento veterinario de cierta entidad. Entiende el autor que la sentencia de la A. Provincial de Valencia se aparta de la línea fijada por el alto tribunal, exigiendo además un inexistente elemento subjetivo específico del injusto consistente en "el sufrimiento animal", que supone una específica carga intencional adicional al dolo.

Palabras claves: bienestar animal; derecho animal; derecho penal; maltrato animal.

Abstract - Analysis of Sentence $n^{\circ}$ 16/21, of 15th January, by the Second Section of the Provincial Hearing in Valencia. The cosmetic cutting of dog ears and its consideration as an animal abuse crime in Spain

This commentary analyses the jurisprudential requirements specified by the concept of severe harm to animal health in the sense required by Supreme Court Judgment 185/20, of 20th May. The essence of these requirements is the need for a certain level of veterinary treatment. The author understands that the ruling of the provincial hearing in Valencia digressed from the line taken by the high court, further demanding an inexistent subjective element specific to unfairness consisting of "animal suffering", which entails a specific intentional charge beyond malice.

Keywords: Animal welfare; animal law; penal law; animal abuse. 


\section{Introducción}

El Juzgado de lo Penal $n^{\circ} 17$ de Valencia, en la Sentencia $n^{\circ} 337 / 20$, de fecha 15/9/20, recaída en el Juicio Oral no 846/2018, condenó a L. L. T., como autor de un delito de intrusismo profesional, del art. 403.1 $\mathrm{CP}$, a la pena de multa de 15 meses con cuota diaria de 10 euros, y por un delito continuado de maltrato animal, de los arts. 337.1 y 3 y 74 CP, a las penas de un año y un día de prisión, accesoria de inhabilitación especial para el ejercicio del derecho de sufragio pasivo durante el tiempo de la condena, e inhabilitación especial de tres años y un día para el ejercicio de profesión, oficio o comercio que tenga relación con los animales.

La citada sentencia contenía un relato de hechos probados, que finalmente se concreta en los siguientes:

“D. L. L. T. se dedicaba, en el año 2014, a la cría de perros en su domicilio, sito en la calle () de la localidad de () (Valencia), desarrollando alli y de manera informal actividades propias del ámbito veterinario, incluidas pequeñas operaciones de cirugía canina, careciendo de la formación y titulación necesaria para realizar este tipo de intervenciones.

Por su parte, D. S. O. C. recibió gratuitamente de su amiga $D^{a} A$. B. B. un cachorro de la raza American Bully, nacido el 27 de diciembre de 2013, y le facilitó el contacto del acusado para que realizara al animal una intervención quirúrgica en las orejas, sin que haya quedado demostrado que el primero conociera la ilicitud de tal operación veterinaria.

El día 12 de marzo de 2014, D. S. O. C. llevó a su perro al domicilio del acusado donde, previa administración de la oportuna anestesia total, este le cortó las orejas. Tal intervención no era necesaria para la salud del animal y precisó puntos de sutura y tratamiento veterinario.

Posteriormente, el día 26 de marzo de 2014, D. S. O. C. regresó con el perro al domicilio del acusado, acompañado de su entonces pareja, Dña. M. F. O. o, con el fin de que el acusado le retirara los puntos de sutura de las orejas. Comoquiera que el animal se movía durante la operación y con el fin de mostrarle autoridad, D. L. L. T. lo cogió del cuello y del lomo y, alzándolo unos veinte centímetros, lo golpeó fuertemente dos veces sobre la mesa de acero inoxidable, quedando el perro aturdido y jadeando.

Como consecuencia de dichos impactos, el perro sufrió una perforación pulmonar con una de sus costillas, lo que le provocó un edema con hemorragia pulmonar masiva. D. S. O. C. llevó al animal a una clínica veterinaria ese mismo día, donde falleció.- El valor de un American Bully oscila entre los 300 y 500 euros, según su estado y características especiales. El coste de la atención veterinaria ascendió a 170 euros.

Asimismo, Dña. M. F. O. sufrió una crisis de ansiedad, requiriendo para sanar diez días no impeditivos. Los perjudicados reclaman por los daños y perjuicios causados.

El presente procedimiento se ha dilatado en exceso por motivos no imputables al acusado, habiendo estado paralizado desde la diligencia de remisión de la causa a este Juzgado de lo Penal el 13 de julio de2018 y hasta el auto de admisión de prueba de fecha 11 de noviembre de 2019, suponiendo tal circunstancia un perjuicio personal para él, que se ha visto sometido a la condición de investigado y acusado más tiempo del razonablemente necesario”.

Contra la indicada sentencia se interpuso, en fecha 25/11/20 recurso de apelación ante la Audiencia Provincial, por parte de la defensa del acusado, solicitando la absolución del mismo. A dicho recurso se adhirió parcialmente el Ministerio Fiscal, por motivos que nada tienen que ver con el contenido del presente artículo, supliendo un error material acerca del tipo penal vigente en el momento de los hechos, solicitando la condena por los dos delitos, si bien solicitando la aplicación, en el delito del art. $337 \mathrm{CP}$, del texto vigente en el año 2014, que lo era el fijado por la LO 5/2010, de 22 de junio.

Las diferentes regulaciones en el texto vigente en el momento de los hechos, y el momento actual, fijado por la LO 1/2015, de 30 de marzo, en nada afectan al presente recurso, pues el motivo de la impugnación se basa en la parte del texto que permanece inalterado de una a otra regulación.

La Sección $2^{\mathrm{a}}$ de la Audiencia Provincial de Valencia, en el Rollo de Apelación de Sentencias de Procedimiento Abreviado $\mathrm{n}^{\mathrm{o}}$ 1604/20, dictó en fecha 15/1/21 la sentencia $\mathrm{n}^{\mathrm{o}} 16 / 21$. En dicha sentencia, se mantiene la condena del acusado por el delito de instrusismo, absolviéndole, en cambio, del delito continuado de maltrato animal. Dicha absolución la basa en una doble argumentación:

En primer lugar, la ausencia de dolo en la conducta del acusado, golpeando en dos ocasiones fuertemente al perro contra la mesa de acero inoxidable (en el relato de hechos, erróneamente, se confunde el nombre del acusado con el del dueño del animal en esta acción, por cierto) y ocasionándole la muerte con ello. 
No se insiste en dicha valoración, aún discrepando de la misma, y estar la misma fuera del ámbito del presente artículo. En segundo lugar, que la operación de corte de orejas del animal no constituye menoscabo grave de la salud del animal, ni tratamiento veterinario; lo cual sí es el objeto del presente artículo.

El delito de maltrato animal que sería aplicable a los hechos es el previsto en el art. 337.1 a) y 3 CP de España:

"1. Será castigado con la pena de tres meses y un día a un año de prisión e inhabilitación especial de un año y un día a tres años para el ejercicio de profesión, oficio o comercio que tenga relación con los animales y para la tenencia de animales, el que por cualquier medio o procedimiento maltrate injustificadamente, causándole lesiones que menoscaben gravemente su salud o sometiéndole a explotación sexual, a

a) un animal doméstico o amansado,

b) un animal de los que habitualmente están domesticados,

c) un animal que temporal o permanentemente vive bajo control humano, o

d) cualquier animal que no viva en estado salvaje. ()

3. Si se hubiera causado la muerte del animal se impondrá una pena de seis a dieciocho meses de prisión e inhabilitación especial de dos a cuatro años para el ejercicio de profesión, oficio o comercio que tenga relación con los animales y para la tenencia de animales".

\section{Legislación aplicable}

El corte de orejas a un perro es una práctica prohibida legalmente. En España, la competencia para la protección animal ha sido asumida por las comunidades autónomas, que han ido legislando sobre esta materia con exclusión casi total de la legislación estatal.

Como excepción al ejercicio de competencia estatal en este ámbito, pueden citarse tres leyes: en primer lugar, la ley 50/1999, de 23 de diciembre, sobre el Régimen Jurídico de la Tenencia de Animales Potencialmente Peligrosos ${ }^{1}$. Esta ley contiene, en su art. 13, dentro del régimen de infracciones y sanciones, una previsión de conducta sancionable donde incluye el abandono del animal como infracción muy grave, sin necesidad de que se produzca resultado alguno. Dicha conducta es susceptible de ser incardinada, con determinadas condiciones, en la figura delictiva del art. 337 bis $\mathrm{CP}$.

En segundo lugar, Ley 8/2003, de 24 de abril, de sanidad animal, aunque la misma prácticamente no recoge más que alguna conducta residual de abandono, en relación a animales de compañía ${ }^{2}$.

En tercer lugar, tenemos la Ley 32/2007, de 7 de noviembre, para el cuidado de los animales, en su explotación, transporte, experimentación y sacrificio ${ }^{3}$. En dicha ley, se contempla, en su Disposición Adicional Primera, la aplicabilidad a los animales de compañía de determinadas disposiciones de la misma; particularmente, el régimen de determinadas infracciones, entre las que se encuentran el sacrificio o muerte de animales, el incumplimiento de obligaciones de cuidado con intención de matar o torturar, o su utilización en peleas.

Así, en la Comunidad Valenciana, que es donde se produjeron los hechos objeto de la sentencia que aquí se analiza, el art. 4.e) de la Ley 4/94, de 8 de julio, de la Generalitat valenciana, sobre protección de los animales de compañía, vigente en el momento de los hechos de la presente causa, establece que:

"se prohibe practicarles mutilaciones (a los animales de compañia), salvo las realizadas por profesionales veterinarios en caso de necesidad justificada. En ningún caso se considerará justificada la estética".

Esta prohibición legal en territorio valenciano, que es de carácter general en la totalidad de las leyes autonómicas de protección animal, se reforzó, si cabe, con la adhesión de España al Convenio del Consejo de Europa sobre protección de los animales de compañía, hecho en Estrasburgo el 13 de noviembre de 1987, por Instrumento de ratificación de 27 de septiembre de 2017, y entrada en vigor el 1 de febrero de 2018, que recoge, en su art. 10.1 b) la prohibición del corte de orejas con fines estéticos. Aunque su entrada en vigor es posterior a los hechos por los que se sigue la presente causa, el mismo viene a subrayar la prohibición citada, ya vigente entonces:

\footnotetext{
${ }^{1}$ BOE núm. 307, de 24 de diciembre de 1999.

${ }^{2}$ BOE núm. 99 de 25 de abril de 2003. En su art. 84.20, recoge como infracción grave "El abandono de animales, de sus cadáveres o de productos o materias primas que entrañen un riesgo sanitario para la sanidad animal, para la salud pública o contaminen el medio ambiente, o su envío a destinos que no estén autorizados, siempre que no esté tipificado como falta muy grave”.

${ }^{3}$ BOE núm. 268 de 08 de Noviembre de 2007.
} 
"1. Se prohibirán las intervenciones quirúrgicas cuyo objeto sea modificar la apariencia de un animal de compañía o conseguir otros fines no curativos $y$, en particular:
a. el corte de la cola;
b. el corte de las orejas;
c. la sección de las cuerdas vocales;
d. la extirpación de uñas y dientes.

2. Sólo se permitirán excepciones a estas prohibiciones:

a. si un veterinario considera necesarias las intervenciones no curativas, bien por razones de medicina veterinaria, o bien en beneficio de un animal determinado;

b. para impedir la reproducción.

3.

a. Las intervenciones en las cuales el animal vaya a sufrir o pueda sufrir dolores intensos sólo podrán efectuarse con anestesia y por un veterinario o bajo su supervisión.

b. Las intervenciones que no requieran anestesia podrán ser efectuadas por una persona competente con arreglo a la legislación nacional”.

Por ello, puede concluirse, sin género de duda, que en la actualidad, el corte de orejas y rabo a un animal, con fines puramente estéticos, es una práctica prohibida en España, y en el resto de países que han ratificado el referido convenio.

\section{Referente jurisprudencial en España}

La referida sentencia de la Audiencia Provincial se ha apartado de la doctrina emanada de la Sala Segunda del Tribunal Supremo, fijada recientemente en la sentencia, de su Sección Primera, 186/2020, de 20 de mayo. Dicha sentencia, al respecto, señala en su fundamento de Derecho SEGUNDO 3 que:

"Una visión integrada del texto penal aconseja una interpretación homogeneizadora. Por ello, a la hora llenar de contenido un concepto valorativo como el de grave menoscabo a la salud, lo más plausible es establecer un parangón con las figuras penales de similares perfiles, en este caso las que protegen la integridad fisica de los humanos: los delitos de lesiones. Esta parece ser la pauta seguida por el legislador a la vista de la similitud en el enunciado de las modalidades agravadas previstas en uno y otro caso. El artículo 337.2 incluye como tales las mismas que los artículos 148 y ss. Tanto aquellas que lo son por la entidad del menoscabo fisico (articulo 149), las que se refieren a los medios comisivos (empleo de instrumentos peligrosos de los artículos 148.1), a los modos de ejecución (el ensañamiento artículos 148.2), o aquellas orientadas a proteger a los menores, como el perpetrar el hecho a su presencia (153.3).

En esta línea, a la hora de concretar lo que deba entenderse por menoscabo grave de la salud al que alude el artículo $337.1 \mathrm{CP}$, un primer enfoque nos proyectaría sobre el concepto de "grave enfermedad" que, cuando de humanos se trata, el artículo 149 equipara a la pérdida o inutilidad de un sentido, órgano, o miembro principal. Sin embargo, tal opción no puede acogerse linealmente porque "la pérdida o inutilidad de un sentido, órgano o miembro principal" están específicamente previstos como presupuestos de agravación en el apartado 2 del artículo 337 que, aun sin sustantividad independiente como ocurre en el delito de lesiones graves del artículo 149, elevan la pena a la mitad superior.

Partiendo de tales premisas la lógica aconseja interpretar la modalidad básica del artículo 337.1 como proyección de su equivalente cuando del delito de lesiones se trata (artículo 147.1), con imprescindibles modulaciones. Tomando como referencia el que se erige como concepto normativo básico en el delito de lesiones, el tratamiento médico o quirúrgico, será necesario que el animal requiera para su curación tratamiento veterinario, más allá del que se agota en una primera asistencia. Ahora bien, ese único presupuesto abarcaría detrimentos de la salud que dificilmente soportarían el calificativo de graves, lo que exige un plus que dependerá de las circunstancias del caso. Este podrá venir determinado por diversos factores. Entre ellos, sin afán de fijar un catálogo exhaustivo, habrán de valorarse la intensidad de la intervención veterinaria requerida; si hubiera exigido o no hospitalización; el riesgo vital generado por la herida o su potencialidad para acelerar significativamente procesos degenerativos; el periodo de tiempo durante el cual el animal haya estado imposibilitado para el desempeño de la actividad propia de su especie; y las secuelas o padecimientos permanentes. Sin olvidar que, si éstos últimos conllevan la pérdida de un sentido, órgano o miembro 
principal, necesariamente determinaran la imposición de la pena en su mitad superior (artículo 337.2). Todo lo que por defecto no tenga encaje en el concepto así perfilado, quedará abarcado por el delito leve del artículo 337.4. CP, que ni siquiera exige que se haya llegado a causar lesión”.

La STS apuntada, que curiosamente menciona la sentencia de la Audiencia que ahora se analiza, señala inequívocamente como requisitos para la existencia de "menoscabo grave de la salud del animal" que exige el tipo, de un lado, el tratamiento médico (veterinario, en este caso), más allá del que se agota en la primera asistencia.

De otro lado, exige valorar "la intensidad de la intervención veterinaria": si el animal ha precisado de hospitalización, el riesgo vital generado por la herida, o su potencialidad para acelerar significativamente procesos degenerativos, el período de tiempo por el que el animal haya estado imposibilitado, y las secuelas o padecimientos permanentes.

Resulta evidente que la citada sentencia trata de diferenciar, y expulsar del precepto, aquellos casos de mínima intervención del veterinario, y que no han significado ni riesgo por la intervención ni la herida, ni excesivo tiempo de curación, ni secuela alguna.

\section{Ajuste de la sentencia analizada a la jurisprudencia española.}

Resulta claro, del relato de hechos probados de la presente sentencia, que el caso que nos ocupa no se encuentra en esta última categoría mencionada, de supuestos de intervención mínima del veterinario. La sentencia impugnada describe los hechos como que "el día 12 de marzo de 2014, D. S. O. C. llevó a su perro al domicilio del acusado donde, previa administración de la oportuna anestesia total, este le cortó las orejas. Dicha intervención no era necesaria para la salud del animal y precisó puntos de sutura y retirada de puntos. Posteriormente, el día 26 de marzo de 2014, D. S. O. C. regresó con el perro al domicilio del acusado () con el fin de que el acusado le retirara los puntos de sutura. Comoquiera que el animal se movía durante la operación...".

En el análisis de esta conducta, la sentencia de la Audiencia Provincial valora dichos hechos del siguiente modo.

"Ciertamente, el legislador reconoció que la mutilación era una forma de maltrato en la ley de 8 de julio de 1994 de la Generalitat Valenciana, de protección e los animales de compañía, cuyo art. 4 e) prohibe expresamente la mutilación de animales y, en concreto, la que se realice con fines estéticos. Solamente se permite por necesidades médicas y realizada por un veterinario, lo que no es el presente caso. Lógicamente, tal como explicó el perito Luis Burillo, el perro debe ser anestesiado y sufre dolor después de la intervención. De otro lado, la aplicación de puntos de sutura supone que después deben ser retirados y con este fin los denunciantes volvieron al domicilio del acusado. Pero, sentado lo anterior, la afirmación de que el acusado produjo un padecimiento grave al animal carece de apoyo probatorio, pues no resulta de ninguna de las pruebas periciales practicadas; y el único tratamiento que objetivamente ha quedado constatado es la retirada de puntos. En concreto, no consta que la operación de corte de orejas alterara la funcionalidad del órgano auditivo ni menoscabara el bienestar animal. En definitiva, los peritos no proporcionaron elementos de juicio o datos que permitan calificar esta operación como de graves consecuencias. Los propios dueños no observaron alteraciones en el perro tras la operación y volvieron confiados para la retirada de puntos. Incluso, el perito de la parte acusadora, Luis Burillo, manifestó que el corte de orejas no tiene complicaciones para la vida del animal. De modo que, con base a la prueba practicada en este juicio, si bien existe un maltrato y un cierto padecimiento, por el dolor postoperatorio, no puede concluirse que el maltrato causara al animal un padecimiento que pueda calificarse de grave. A la vista de la prueba pericial, tampoco puede afirmarse la necesidad objetiva de tratamiento veterinario, más allá de la lógica retirada de puntos".

Se reitera que no se discute la valoración de la prueba, por más que la misma no se ajuste, exactamente, al contenido de lo acaecido en la vista oral, ciñéndose por completo a la valoración que se acaba de transcribir.

Resulta evidente que se deja de aplicar, indebidamente, según lo expuesto, el art. $337 \mathrm{CP}$, en su redacción establecida por la LO 5/201, de 22 de junio, que exige la causación de "menoscabo grave de la salud del animal". Ello, por los siguientes argumentos:

A. Aunque pudiera deducirse de una interpretación hermenéutica del precepto, teniendo en cuenta el bien jurídico protegido del bienestar animal, "el padecimiento grave" del mismo no es elemento del tipo penal, como señala erróneamente la sentencia de instancia. De hecho, el delito 
podría, perfectamente cometerse sin tal padecimiento.

B. En este sentido, la doctrina más reciente, a cuyo frente se encuentra HAVA GARCÍA resalta que lo que protege el delito es el propio bienestar animal, y no los sentimientos de las personas ante el mismo ${ }^{4}$. En el mismo sentido, CUERDA ARNAU incide en que la reforma operada por la LO $1 / 2015$, deja claro que el bien jurídico protegido es "el bienestar animal vinculado a una ausencia de sufrimiento innecesario". Subraya la autora, como ya veíamos, que las sucesivas reformas de los preceptos legales han ido aclarando la cuestión, que ha ido oscilando desde los intereses generales que señalaban las antiguas faltas del art. $631 \mathrm{CP}$, pasando por los sentimientos de las personas, hasta el propio animal ${ }^{5}$.

También BRAGE CENDÁN considera como tal al bienestar animal "entendido como derecho de ciertos animales a no sufrir un maltrato innecesario e injustificado". En igual sentido, otros autores que hablan del bienestar animal, lo que incluye su vida e integridad, incluida la psíquica, o el propio animal, en sí mismo considerado ${ }^{7}$. Finalmente, como una evolución de las anteriores doctrinas, algunos autores hablan de atribuir a los animales la titularidad de derechos subjetivos ${ }^{8}$.

Jurisprudencialmente, por su parte, la postura mayoritaria se inclina por considerar, explícitamente, como el bien jurídico protegido por estos delitos la dignidad del animal como ser vivo .

Finalmente, la reforma operada por la LO 1/2015, al diferenciar, penológicamente, las conductas de causación de lesiones, y las de muerte del animal, viene a dar la razón a los defensores de estas posturas doctrinales, al regularse estas conductas de modo semejante al utilizado para las lesiones y la muerte de seres humanos. Así, RÍOS CORBACHO, señala que no es que el animal no posea derechos subjetivos, sino que los mismos no pueden colocarse en el mismo plano que los de los seres humanos ${ }^{10}$.

Toda la doctrina citada vincula, de algún modo, el bien jurídico del bienestar animal, o de la dignidad del animal, a la causación de un sufrimiento innecesario del animal, pero no a la presencia de un específico elemento subjetivo del injusto consistente en la voluntad de querer causar dicho sufrimiento; bastando con la aceptación del hecho de que se va a producir el mismo, y que la conducta no está justificada. Como sería, por ejemplo, con una operación realizada por un veterinario por motivos sanitarios justificados, no por una operación estética realizada por un no profesional, como el caso que nos ocupa.

Este inexistente elemento subjetivo específico del injusto consistente en "el sufrimiento animal" supone una específica carga intencional adicional al dolo. Dicho requisito podría parecer, en abstracto, acorde con el bien jurídico protegido y las condiciones que el tipo exige para esta protección: se protege el bienestar animal en las condiciones en el que éste convive con el hombre. Así, lo protegido no es la vida e integridad del animal, sino su bienestar, o ausencia de sufrimiento, por lo que sólo las conductas enfocadas a perturbar este bienestar, o a causar sufrimiento, deberían se antijurídicas.

\footnotetext{
${ }^{4}$ Señala la autora que "la protección penal que se le otorga en nuestro país al paisaje o al patrimonio histórico-artístico está inspirada por los sentimientos humanos que suscita la contemplación de la belleza en sus diversas formas, pero ello no obliga a afirmar que los bienes jurídicos protegidos en los tipos penales que se encargan de su tutela sean tales sentimientos ( ) tanto la belleza artística como el patrimonio histórico satisfacen necesidades humanas y que, por tanto, constituyen auténticos bienes jurídicos de carácter colectivo". HAVA GARCÍA, E. La tutela penal de los animales. Tirant lo Blanch. (Valencia 2009). Págs. 123 y 124.

${ }^{5}$ CUERDA ARNAU, M.L. Maltrato y abandono de animales, en Comentarios a la reforma del Código Penal de 2015 (González Cussac, J.L. director). $2^{\mathrm{a}}$ edición. Tirant lo Blanch (Valencia 2015).

${ }^{6}$ BRAGE CENDÁN, S. Los delitos de maltrato y abandono de animales (artículos 337 y 337 bis CP). Tirant lo Blanch (Valencia 2017) Pág. 59.

${ }^{7}$ REQUEJO CONDE, C. El delito de maltrato a los animales tras la reforma del Código Penal por Ley Orgánica 1/2015, de 30 de marzo, en dA. Derecho Animal (Forum of Animal Law Studies) 6/2 (2015). Págs. 9 y 10. DOI: https://doi.org/10.5565/rev/da.77

DELGADO GIL razona que "el delito lo que protege es la salud y vida misma de los animales en sí mismas consideradas". DELGADO GIL, A. Antecedentes y bien jurídico protegido en los delitos de maltrato animal, en La ley penal 123 (noviembrediciembre 2016). 1 a 9. Págs. 5 y 6.

${ }^{8}$ PELAYO GONZÁLEZ-TORRE, A. Sobre los derechos de los animales, en Anuario de Filosofía del Derecho, VII (1990). Pág. 553.

${ }^{9}$ Este concepto, que se razona como que "El bien jurídico protegido es la dignidad del animal como ser vivo que debe prevalecer, cuando no hay un beneficio legítimo en su menoscabo que justifique su sufrimiento gratuito”, se acuño en la SAP Madrid, Sec. 17", $\mathrm{n}^{\circ}$ 287/2004, de 19 de abril, (TOL497.659), y ha sido reproducido, literalmente, en infinidad de sentencias desde entonces. Por ejemplo, SAP Barcelona, Secc. 9a, no 382/2007, de 24 de octubre (TOL1.231.223), AP Vizcaya, sec. $1^{\mathrm{a}}$, no ${ }^{\mathrm{o}} 574 / 2006$, de 25 de octubre (TOL1.061.503), SAP Pontevedra, Sec. $4^{\mathrm{a}}, \mathrm{n}^{\mathrm{o}}$ 116/08, de 5 de junio (TOL7.024.053), AP Lleida, sec. $1^{\mathrm{a}}, \mathrm{n}^{\mathrm{o}}$ 93/2008, de 3 de marzo (TOL1.303.295), AP Madrid, sec. $2^{\mathrm{a}}$, $\mathrm{n}^{\mathrm{o}} 824 / 2015$, de 5 de octubre, (TOL5.578.500), Juzgado de lo Penal $\mathrm{n}^{\circ} 3$ de Santander, $\mathrm{n}^{\circ} 72 / 2016$, de 30 de marzo (TOL5.681.426).

${ }^{10}$ RÍOS CORBACHO, J.M. Nuevos tiempos para el delito de maltrato de animales a la luz de la reforma del código penal español (LO 1/2015), en Revista Electrónica de Ciencia Penal y Criminología (2016). Pág. 22.
} 
CUERDA ARNAU niega expresamente este requisito ${ }^{11}$. Entiende, en primer lugar, que el principio de legalidad hubiera exigido que así se explicitara en el tipo, de modo similar a otros muchos preceptos del $\mathrm{CP}^{12}$. En segundo lugar, porque, correlativamente a lo anterior, el propio legislador tiende a una evolución del precepto, desde 1995, en el sentido de objetivizar el precepto, eliminando expresiones que figuraban en el mismo, como requisitos tendenciales, dentro del mismo. Tal es el caso de los términos cruel y ensañamiento, que hoy no figuran en este tipo básico y, en su caso, sirven de tipo residual o para la agravación del subtipo, respectivamente.

En definitiva, se entiende que se trata de una restricción teleológica, aunque arbitraria, del tipo analizado, sin base legal. Lo que debe preguntarse el Tribunal es si la conducta del autor se encuentra justificada, o no, y si tal conducta atenta a la dignidad del animal, con una causación innecesaria de sufrimiento. Por supuesto, tampoco permite el tipo ninguna interpretación parecida a lo que señala la sentencia de la Audiencia Provincial, ahora recurrida, exigiendo, además, que ese sufrimiento sea grave.

En todo caso, dicho padecimiento o sufrimiento existe; no hay que olvidar que la intervención realizada supone la mutilación de la mitad de cada una de las dos orejas del animal, aplicación de puntos de sutura (aunque no se especifica, la propia descripción de los hechos permite aventurar una gran cantidad de los mismos, dado que el animal contaba ya con tres meses de edad; no siendo recién nacido). Dichos puntos los lleva el animal durante 14 días, nuevamente es situado en la mesa de operaciones, donde se le retiran, especificando la sentencia que el animal se movía durante la operación, dificultando notoriamente la misma, hasta el punto de que el acusado tuvo que golpear fuertemente al animal durante dicha retirada de puntos. El animal, además, quedó notoriamente mutilado tras la operación.

Parece olvidar también la sentencia analizada que esta acción de golpear fuertemente al animal contra la mesa de acero inoxidable, por dos ocasiones, lo que, a pesar de que lo considera no doloso, sin duda alguna causa un grave padecimiento al animal.

Es que además, en el relato de hechos probados que fija la Audiencia Provincial, se recoge expresamente que "quedando el perro aturdido y jadeando. Como consecuencia de dichos impactos, el perro sufrió una perforación pulmonar con una de sus costillas, lo que le provocó un edema con hemorragia pulmonar masiva". Se estima pues que, en esta parte del relato de hechos probados, en la segunda fase de la operación del corte de orejas, la retirada de puntos, la Audiencia concreta hechos que denotan con claridad dicho sufrimiento.

C. Considera la sentencia que no puede afirmarse la necesidad objetiva de tratamiento veterinario. Se discrepa radicalmente de tal afirmación. Así, en primer lugar, existió una anestesia general para la intervención. Ello, en aplicación de la STS 186/20, de constante referencia, lo equipararía a intervención quirúrgica con hospitalización, de haberse dado en seres humanos. Sin duda, la aplicación de anestesia general, indicada en estos casos, supone un tratamiento veterinario.

En segundo lugar, se le aplicó el animal un corte en cada una de sus dos orejas que seccionó el pabellón auricular y el cartílago de cada una de ellas. Esto supone el corte de hasta tres arterias de cada una de las orejas, que precisan de cierre inmediato, mediante puntos, para evitar la muerte por desangrado del animal, lo que se hizo.

En tercer lugar, existió una segunda intervención, a los 14 días, para la retirada de los puntos con material quirúrgico.

D. Que el animal no haya precisado hospitalización no deriva más que de la dinámica delictiva del acusado, quien ha sido condenado por intrusismo, lo que lógicamente le excluye del sistema sanitario de clínicas veterinarias que, en supuestos como el presente, aconseja la permanencia en clínica (hospitalización, pues), durante las primeras horas posteriores a la doble operación.

E. La potencialidad del riesgo de la operación resulta palmaria, por el corte con sangrado, que provoca la necesidad de sutura inmediata de ambos pabellones auriculares.

Esto, si cabe, se acentúa en el presente caso por la circunstancia relevante de que el acusado no es veterinario, con lo que el riesgo para la vida e integridad física del animal se multiplica.

\footnotetext{
${ }^{11}$ CUERDA ARNAU, M.L. en “Comentarios a la...”. Cit. Pág. 1088.

${ }^{12}$ Por ejemplo, en el delito de hurto del art. $234 \mathrm{CP}$, se exige "ánimo de lucro". Otras veces, se define el tipo con un elemento subjetivo de manera negativa; es decir, se realiza el tipo, siempre que no se realice con un ánimo específico (en el delito de uso de vehículo de motor ajeno del art. $244 \mathrm{CP}$, que no se realice con el ánimo de apropiárselo, porque, caso contrario, sería un delito de hurto o robo de los arts. 234 ó $237 \mathrm{CP}$ ). Además, jurisprudencialmente se recurre a elementos subjetivos en ocasiones que el legislador no ha previsto expresamente o, al menos, ello es dudoso (p.ej. art. 525.2 CP, en relación al art. 525.2 CP).
} 
F. El animal permaneció 14 días con los puntos, y las secuelas de la intervención son evidentes: le priva de la mitad de sus dos orejas durante el resto de su vida. Aunque es cierto que la sentencia de instancia razona que ello no menoscaba la funcionalidad del órgano auditivo, la totalidad de la literatura científica actual contradice tal sorprendente afirmación, si bien ello no es objeto del presente recurso.

Por ello, cada uno de los requisitos exigidos por la mencionada STS 186/2020, se cumplen en el presente caso:

a) Existió un tratamiento veterinario constituido por la anestesia general, corte de orejas, puntos de sutura y posterior retirada de los mismos a los 14 días. Dicha intervención quirúrgica supone un menoscabo grave de la salud el animal, por resultar prohibida e innecesaria. Solo hubiera estado justificada por razones de salud.

b) La intensidad de la intervención veterinaria no es de carácter leve, por lo expuesto; particularmente la alteración anatómica producida al animal, las características de la operación, el padecimiento sufrido y el innecesario riesgo que, para la vida e integridad del animal, supuso al ser realizada además, por un lego.

El animal resultó con una evidente alteración anatómica de las dos orejas que le restó como secuela tras la operación (hasta que murió, tras ser golpeado contra la mesa donde la misma se desarrolló).

Subsidiariamente, tal y como recoge la STS 168/20, el tribunal, si no consideraba que lo relatado constituía tratamiento médico-veterinario, o el mismo era de carácter insignificante, debió condenar por el maltrato cruel recogido en el art. 337.4 CP (anterior art. 632.2 CP, en la redacción vigente en el momento de los hechos $)^{13}$.

Dicha sentencia señala, al respecto, que "El tipo que nos ocupa se encuentra en relación de subsidiariedad expresa con los que le preceden en el artículo 337. Así señala "fuera de los supuestos a que se refieren los apartados anteriores de este articulo, maltrataren cruelmente...". Es decir, quedan fuera de su ámbito de aplicación aquellos casos en que, a consecuencia del cruel maltrato, se causare la muerte del animal (artículo 337.3) o lesiones que menoscaben gravemente su salud (artículo 337.1). () Todo lo que por defecto no tenga encaje en el concepto así perfilado (art. $337.1 \mathrm{CP}$ ), quedará abarcado por el delito leve del artículo 337.4. CP , que ni siquiera exige que se haya llegado a causar lesión".

\section{Conclusiones}

1. El corte estético de orejas y rabo a animales es una práctica prohibida en España, a tenor de la legislación analizada, salvo que sean realizadas por motivos curativos.

2. Dichas operaciones estéticas causan un gran sufrimiento al animal, y precisan, para su realización, de tratamiento veterinario de cierta entidad. Dicho tratamiento es considerado como lesiones que menoscaban gravemente la salud del animal.

3. La STS 186/2020, de 20 de mayo, avala plenamente esta anterior interpretación.

4. La Sentencia $n^{0} 16 / 21$, de 15 de enero, de la Sección Segunda de la Audiencia Provincial de Valencia, se aparta notoriamente de la línea jurisprudencial apuntada, dejando de aplicar sus líneas fundamentales, y exigiendo un inexistente elemento subjetivo específico del injusto, como es el sufrimiento o padecimiento animal; si bien es cierto que el bien jurídico protegido en la figura delictiva obliga a una interpretación integradora en el sentido apuntado anteriormente. Dicho padecimiento existe en las operaciones estéticas analizadas, en todo caso.

\section{Legislación}

- Ley 50/1999, de 23 de diciembre, sobre el Régimen Jurídico de la Tenencia de Animales Potencialmente Peligrosos.

- Ley $8 / 2003$, de 24 de abril, de sanidad animal.

- Ley 32/2007, de 7 de noviembre, para el cuidado de los animales, en su explotación, transporte, experimentación y sacrificio.

\footnotetext{
13 "Los que maltrataren cruelmente a los animales domésticos o a cualesquiera otros en espectáculos no autorizados legalmente sin incurrir en los supuestos previstos en el artículo 337 serán castigados con la pena de multa de 20 a 60 dias o trabajos en beneficio de la comunidad de 20 a 30 días".
}

206 Derecho Animal. Forum of Animal Law Studies, vol. 12/2 
- Ley 4/94, de 8 de julio, de la Generalitat valenciana, sobre protección de los animales de compañía-

- Convenio del Consejo de Europa sobre protección de los animales de compañía, hecho en Estrasburgo el 13 de noviembre de 1987. Instrumento de ratificación el Reino de España, de 27 de septiembre de 2017.

- Ley Orgánica 10/1995, de 23 de noviembre, del Código Penal.

\section{Bibliografía}

- BRAGE CENDÁN, S. "Los delitos de maltrato y abandono de animales (artículos 337 y 337 bis CP)". Tirant lo Blanch (Valencia 2017)

- CUERDA ARNAU, M.L. Maltrato y abandono de animales, en Comentarios a la reforma del Código Penal de 2015 (González Cussac, J.L. director). $2^{\mathrm{a}}$ edición. Tirant lo Blanch (Valencia 2015).

- DELGADO GIL, A. Antecedentes y bien jurídico protegido en los delitos de maltrato animal, en La ley penal 123 (noviembre-diciembre 2016).

- HAVA GARCÍA, E. La tutela penal de los animales. Tirant lo Blanch (Valencia. 2009).

- PELAYO GONZÁlEZ-TORRE, A. Sobre los derechos de los animales, en Anuario de Filosofía del Derecho, VII (1990). Pág. 553.

- REQUEJO CONDE, C. El delito de maltrato a los animales tras la reforma del Código Penal por Ley Orgánica 1/2015, de 30 de marzo, en dA. Derecho animal (Forum of Animal Law Studies) 6/2/ (2015). DOI: https://doi.org/10.5565/rev/da.77. Págs. 9 y 10.

- RÍOS CORBACHO, J.M. Nuevos tiempos para el delito de maltrato de animales a la luz de la reforma del código penal español (LO 1/2015), en Revista Electrónica de Ciencia Penal y Criminología. 2016. 